\title{
Didaktische Modellierung einer Service-Learning-Lehrveranstaltung - Ergebnisse eines Design-Based-Research-Ansatzes
}

\author{
Karina Fernandez $\cdot$ Peter Slepcevic-Zach
}

Online publiziert: 9. Januar 2018

(C) Der/die Autor(en) 2018. Dieser Artikel ist eine Open-Access-Publikation.

Zusammenfassung Die Einführung einer Service-Learning-Lehrveranstaltung im Rahmen des Masterstudiums Wirtschaftspädagogik stellt den Ausgangspunkt der hier vorgestellten Untersuchung dar. Obwohl bereits einige Studien zur Wirksamkeit von Service-Learning-Veranstaltungen bestehen, sind die Fragen der didaktischen Modellierung einer solchen sowie die sich daraus ergebenden Wirkungen noch wenig beachtet. Ziel dieser Studie war es, das Lehrveranstaltungskonzept Service-Learning im Rahmen des Studiums der Wirtschaftspädagogik zu evaluieren und in seiner didaktischen Modellierung weiterentwickeln.

Als Methode wurde ein Design-Based-Research-Ansatz gewählt. Die Studierenden $(N=82)$ wurden dabei über vier Semester mit Hilfe einer quantitativen Pre-PostFragebogenerhebung (inklusive einer Kontrollgruppe aus dem Masterstudium Betriebswirtschaft, $N=105$ ) sowie unterschiedlichen qualitativen Erhebungsformaten befragt.

Es konnten einerseits die Wirksamkeit dieser Service-Learning-Veranstaltung belegt und andererseits für die didaktische Modellierung fünf Gestaltungselemente identifiziert werden. Zentral für die Wirksamkeit ist eine offene Problemstellung, welche die Lernenden in einer Situation der Unsicherheit belässt, sowie die Lernenden in Kontakt mit den KlientInnen der sozialen Organisationen zu bringen.

Schlüsselwörter Service-Learning · Design-Based-Research · Didaktische Modellierung

Mag. Dr. K. Fernandez $(\bowtie) \cdot$ Ass.-Prof. Mag. Dr. P. Slepcevic-Zach $(\bowtie)$

Institut für Wirtschaftspädagogik, Karl-Franzens-Universität Graz, Universitätsstraße

15/G1, 8010 Graz, Österreich

E-Mail: karina.fernandez@uni-graz.at; peter.slepcevic@uni-graz.at 


\title{
Structuring the teaching and learning processes for a Service-Learning course - Results of an Design-Based-Research Approach
}

\begin{abstract}
The starting point of the presented study was the implementation of a service-learning course in the master program business education and development. Although several studies proof the effectiveness of service-learning, aspects regarding the instructional design are less emphasized in past research. The focus of this study is to evaluate the concept of service-learning und develop the instructional design.

The chosen method was a design-based-research approach including a quantitative pre-post questionnaire survey (with a control group from the Master of Business Administration) and several qualitative surveys. The study was undertaken over a period of four semesters.

The collected and analyzed data prove the effectiveness of the investigated service-learning course. Additionally five design variables for didactic modeling were identified. It appears to be central for effectiveness to confront the students with an undefined problem, which leaves them in a situation of uncertainty. Secondly it is important to establish contact between the students and the clients of the social organizations.
\end{abstract}

Keywords Service-Learning $\cdot$ Design-Based-Research $\cdot$ Structuring teaching and learning processes

\section{Einleitung}

Eine zentrale Aufgabe der Universität ist neben der Forschung die Lehre. Den Lehrenden fällt die Aufgabe zu, die Kompetenzentwicklung der Studierenden zu fördern und zu fordern. Gleichzeitig wird von den Hochschulen gesellschaftliches Engagement erwartet. Bereits Dewey (1915) führte aus, dass die Sensibilisierung für gesellschaftliches Engagement in den universitären Bildungsprozessen ein Ziel darstellt. Dies deckt sich sowohl mit dem Ziel der Universität, die Studierenden in ihrer Persönlichkeitsentwicklung zu unterstützen bzw. diese zur Teilhabe am gesellschaftlichen Leben generell zu befähigen (Euler 2005), als auch mit der Auffassung von Bildung ,als Selbstbestimmungs- und Mitbestimmungsfähigkeit des Einzelnen und als Solidaritätsfähigkeit“ (Klafki 1985, S. 17). Die Umsetzung dieser Ansprüche bedarf entsprechender Lehr-Lernformate. Service-Learning versucht, den zentralen Aufgaben der Universität mit einer entsprechenden didaktischen Modellierung gerecht zu werden.

Dieser Beitrag untersucht mit Hilfe eines Design-Based-Research-Ansatzes die Einführung einer Service-Learning-Veranstaltung im Rahmen des Masterstudiums Wirtschaftspädagogik an der Karl-Franzens-Universität Graz. Die Sensibilisierung für gesellschaftliche Entwicklungen ist gerade für Studierende der Wirtschaftspädagogik von Bedeutung, da diese die Möglichkeit haben, in den Lehrberuf zu gehen. Die Studie wurde dabei über vier Semester mit Hilfe einer quantitativen Pre-PostFragebogenerhebung (inklusive einer Kontrollgruppe aus dem Masterstudium Be- 
triebswirtschaft) sowie unterschiedlichen qualitativen Erhebungsformaten durchgeführt. Die Forschungsfragen der Studie sind:

Wie muss die didaktische Modellierung dieser konkreten Lehrveranstaltung gestaltet sein, um eine möglichst lernprozessförderliche Umgebung zu gewährleisten?

Welche Wirkungen ergeben sich bei den Lernenden durch die Lehr-Lernform Service-Learning?

\section{Theoretischer Hintergrund und Gestaltung von Service-Learning}

\subsection{Definition und Entwicklung}

Service-Learning ist ein Veranstaltungsformat, bei dem Inhalte des jeweiligen Studiums mit gemeinnützigem Engagement der Studierenden verbunden werden; dabei kommen Elemente des erfahrungs-, problem- bzw. handlungsorientierten Lernens zum Einsatz (Deeley 2010). Das Ziel dieses Veranstaltungsformats besteht darin, dass es den Studierenden gelingt, über die Reflexion der gemachten Erfahrungen ein elaboriertes Verständnis der Studieninhalte zu erreichen. Des Weiteren soll bei den Lernenden eine Sensibilisierung zur Frage der eigenen Position hinsichtlich des gesellschaftlichen Engagements angestoßen werden (Slepcevic-Zach und Gerholz 2015).

Im US-amerikanischen Raum ist dieses Format weit verbreitet, es hat sich aber auch in der europäischen Bildungsdiskussion in den letzten Jahren etabliert (Backhaus-Maul und Roth 2013). Im deutschsprachigen Bereich finden sich in Schulen seit den 1990er-Jahren Ansätze von Service-Learning in unterschiedlichen Ausformungen (Sliwka und Frank 2004); die Hochschulen folgten ungefähr ein Jahrzehnt später (Bartsch 2009). Für Österreich muss gesagt werden, dass es erst sehr wenige Initiativen zur Einführung von Service-Learning sowohl an der Schule als auch an der Hochschule gibt. Die Wirksamkeit von Service-Learning-Arrangements ist vor allem durch angelsächsische Studien umfassend untersucht worden (als Überblick Slepcevic-Zach und Gerholz 2015). Die Studien zeigen, dass durch ServiceLearning-Formate sowohl der subjektive Lernerfolg der Studierenden (Reinders und Wittek 2009), ihr Selbstbild (Astin et al. 2000) im Sinne der Erweiterung personaler Einsichten, ihr Verständnis für soziale Herausforderungen bzw. ihr zivilgesellschaftliches Engagement (Arney 2006) sowie ihre Engagementbereitschaft (Mabry 1998) positiv beeinflusst werden. Widersprüchliche Ergebnisse finden sich hinsichtlich der Frage, ob es Unterschiede im Kompetenzaufbau gibt (Astin et al. 2000; Boss 1994). Für den deutschsprachigen Bereich finden sich im Vergleich dazu erst wenige Studien zum Service-Learning. Insbesondere die Wirkungen und mögliche didaktische Modellierungen passend zur Bildungstradition der deutschsprachigen Universitäten sind kaum Gegenstand von Studien gewesen.

\subsection{Didaktische Modellierung von Service-Learning}

Service-Learning kann ein mögliches Format für die hochschulische Bildung sein, welches das Lernen der Studierenden in den unterschiedlichsten Bereichen fördert 
Abb. 1 Didaktische Modellierung von Service-Learning. (Gerholz und Losch 2015, S. 608)

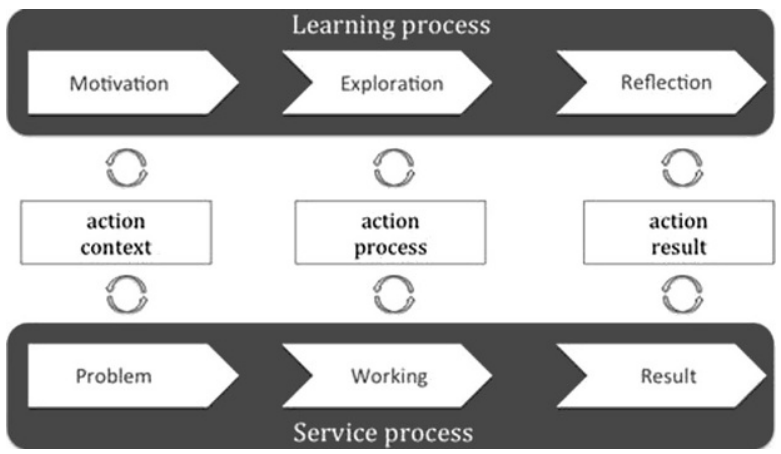

und fordert und ein zivilgesellschaftliches Engagement bei den Lernenden anregt. Für die Konzeption der in diesem Beitrag untersuchten Lehrveranstaltung wurde auf der Definition von Bringle und Clayton aufgebaut, die Service-Learning als ,,competency-based, credit-bearing educational experience in which students (a) participate in mutually identified service activities that benefit the community, and (b) reflect on the service activity in such a way as to gain further understanding of course content, a broader appreciation of the discipline, and an enhanced sense of personal values and civic responsibility“ (2012, S. 105) sehen.

Durch den Anspruch, den Lernprozess der Studierenden mit Problemstellungen sozialer Organisationen zu verbinden, ergeben sich besondere Anforderungen an die didaktische Modellierung von Service-Learning-Formaten. Zentral sind dabei, wie Godfrey et al. (2005) herausstreichen, die Elemente Realität, Reflexion und Gegenseitigkeit. Es muss sich um eine reale Problemstellung der Gesellschaft handeln, die möglichst unterschiedliche soziale Herausforderungen (z. B. Armut, Arbeitslosigkeit, soziale Benachteiligung) widerspiegelt. Die bei der Bearbeitung der Problemstellung erlebte Handlungswirksamkeit muss reflektiert werden (Dewey 1966) und eine partnerschaftliche Lernerfahrung von Studierenden und sozialen Organisationen soll erzeugt werden. Bei der didaktischen Modellierung müssen somit der Lern- und Serviceprozess miteinander verknüpft werden (siehe Abb. 1).

Der handlungstheoretischen Fundierung (Buschfeld 2003) von Service-LearningFormaten folgend sind dabei die Handlungssituation (d.h. die konkrete Problemstellung, die beteiligten Personen sowie die zur Verfügung stehenden Informationen), der Handlungsprozess (d.h. die Aktivitäten, die Aktualisierung bezogen auf das Vorwissen und die Reflexion sowie die Rückmeldung) und das Handlungsergebnis (d.h. die Präsentation der Problemlösung, die Dokumentation sowie die Ergebniskontrolle) zu beachten.

\subsection{Umsetzung im Masterstudium Wirtschaftspädagogik an der Karl- Franzens-Universität Graz}

In der Diskussion um die Einführung von Service-Learning-Formaten für eine wissenschaftliche Berufsvorbildung schwingt immer die Frage nach dem gesellschaftlichen Engagement von Hochschulen mit, d.h. inwiefern soll die Sensibilisierung 
für gesellschaftliches Engagement in (universitären) Bildungsprozessen ein Ziel darstellen? (Jacoby 2009) Das Masterstudium der Wirtschaftspädagogik ${ }^{1}$ verfolgt u.a. das Ziel der Förderung einer wissenschaftlich basierten Handlungskompetenz. Dazu gehört beispielsweise die Herausbildung von Problemlösefähigkeit bei den Studierenden, die es ihnen ermöglicht, Probleme in ihren zukünftigen Handlungsfeldern zu erkennen und mithilfe von wissenschaftlichen Verfahren zu bearbeiten bzw. zu lösen. (Slepcevic-Zach 2017) Bezogen auf den beruflichen Bereich lässt sich das Ziel des Studiums als Förderung beruflicher Handlungskompetenz verallgemeinern und beispielsweise über die Dimensionen einer Fach-, Methoden-, Sozial- und Selbstkompetenz (Peterßen 2009) darstellen. Die Selbst- bzw. Humankompetenz zielt dabei u.a. auf die Fähigkeit der Lernenden ab, ihr eigenes Handeln in Abstimmung mit gesellschaftlichen Wertvorstellungen verantwortungsvoll zu gestalten und ein eigenes Selbstbild zu entwickeln (Reetz 1999). Dieser Prozess benötigt vor allem die Fähigkeit zur Reflexion, welche ganz generell für die Entwicklung der wirtschaftspädagogischen Professionalität von großer Bedeutung ist. Die Auswirkung der angesprochenen polyvalenten Studienstruktur wird daran ersichtlich, dass die AbsolventInnen in einem breiten Spektrum an Beschäftigungsfeldern gefunden werden. Befragt nach der derzeitigen Berufstätigkeit zeigt sich, dass ungefähr $30 \%$ in der Schule, $60 \%$ in verschiedenen Bereichen der Wirtschaft und die restlichen $10 \%$ in den Bereichen Hochschulen/Forschung und Erwachsenenbildung zu finden sind (Zehetner et al. 2016).

Aus einer wirtschaftspädagogischen Perspektive heraus kann Service-Learning somit ein Format sein, welches die Förderung einer wissenschaftlichen und beruflichen Handlungskompetenz bei den Lernenden in bestimmten Bereichen unterstützt. Die in diesem Beitrag untersuchte Lehrveranstaltung ist im fünften und letzten Semester des Masterstudiums Wirtschaftspädagogik angesiedelt. Die Studierenden arbeiten dabei typischerweise in Vierergruppen jeweils mit einer sozialen Organisation zusammen. Die Projektaufträge werden vor Beginn der Lehrveranstaltung mit den Organisationen besprochen, die jeweils eine Ansprechperson für die Studierenden zur Verfügung stellen.

Anhand der angeführten Überlegungen zur didaktischen Modellierung wurde der Ablauf der sich über ein Semester erstreckenden Lehrveranstaltung folgendermaßen konzipiert:

- Einheit 1: Vorstellung der Projekte zusammen mit den sozialen Organisationen

- Einheit 2: Die Studierenden skizzieren ihre ersten Erfahrungen mit der Organisation und ihren Projektauftrag

- Einheit 3, 4 und 5: Zwischenpräsentation der Projektfortschritte (Handlungsprozess und -ergebnis)

- Einheit 6: Probepräsentationen

\footnotetext{
1 Auch wenn in historischer, programmatischer und wissenschaftspolitischer Hinsicht viele Gemeinsamkeiten zwischen der deutschen und österreichischen Wirtschaftspädagogik bestehen (Tafner et al. 2013), muss hier auf einige österreichische Besonderheiten verwiesen werden: Die wissenschaftliche Berufsvorbildung durch das Masterstudium Wirtschaftspädagogik ist polyvalent, folgt einer einphasigen Studienstruktur und ist nicht kombinationspflichtig bzw. -fähig (Slepcevic und Stock 2009).
} 
Tab. 1 Service-Learning Projekte

\begin{tabular}{llll}
\hline $\mathrm{Nr}$ & Semester & Organisation (Problemstellung) & Projektziel \\
\hline 1 & WS & $\begin{array}{l}\text { Pro Mente Steiermark (Wieder- } \\
\text { einstieg von psychisch kranken } \\
\text { Personen in den Arbeitsmarkt) }\end{array}$ & $\begin{array}{l}\text { Entwicklung eines Marketingkonzepts } \\
\text { für die Vitamintankstelle inklusive } \\
\text { Verkaufstraining für die KlientInnen }\end{array}$ \\
& \multirow{2}{*}{ SoSe 2015 } & $\begin{array}{l}\text { Elisabeth-Heim (Therapie- und Be- } \\
\text { treuungsmöglichkeiten für schwerst- } \\
\text { behinderte Menschen) }\end{array}$ & $\begin{array}{l}\text { Erarbeitung und Implementierung (inkl. } \\
\text { Einschulung) eines Kostenrechnungs- } \\
\text { systems inkl. Abrechnungsvorlage für } \\
\text { die Fördergeber }\end{array}$ \\
& WS & $\begin{array}{l}\text { Arbeitskreis Leoben bestehend aus } \\
\text { Vinziwerke, Gemeinde, Pfarre und } \\
\text { Caritas (Leoben bietet keine Not- }\end{array}$ & $\begin{array}{l}\text { Bedarfserhebung einer Notschlafstel- } \\
\text { le in Leoben sowie Erstellung eines }\end{array}$ \\
& Finanzplans für deren Betrieb \\
& Schlafstelle an) & Forum Wohnen (Zusammenschluss \\
& SoSe 2016 & $\begin{array}{l}\text { Kostenanalyse der unterschiedlichen } \\
\text { ainrichtungen und Identifizieren neuer }\end{array}$ \\
& tungen) & $\begin{array}{l}\text { Lösungswege zur Erstbezugsfinanzie- } \\
\text { rung }\end{array}$ \\
& &
\end{tabular}

- Einheit 7: Öffentliche Abschlusspräsentation

- Einheit 8: Reflexionseinheit

Um eine Vorstellung über die Projekte zu bekommen, sind in Tab. 1 vier Projekte aus den vier Semestern (in Summe wurden 18 Projekte durchgeführt) aufgelistet. Dabei werden kurz die Organisation bzw. die Problemstellung sowie das jeweilige Projektziel skizziert. Für das Studium der Wirtschaftspädagogik am Standort Graz eignen sich durch die polyvalente Studienstruktur bzw. mit Blick auf die Beschäftigungsfelder der AbsolventInnen sowohl Projekte mit einem betriebswirtschaftlichen Hintergrund als auch solche, die sich mit dem Lernen und Lehren in und mit Organisationen auseinandersetzen.

Die Studierenden bringen ihre bisher erworbenen Kompetenzen in die sozialen Organisationen ein und arbeiten gemeinsam an der Lösung des jeweiligen Problems. In diesem Setting entsteht die Situation, dass es keine Musterlösung oder ähnliches für das Problem geben kann; die Arbeit wird deswegen von einem laufenden PlanDo-Check-Act-Zyklus (Deming 1989) begleitet. Im gesamten Prozess, insbesondere in den Reflexionsphasen, wird zwischen diesem Service- und dem Lernergebnis unterschieden. Das Lernergebnis zielt auf die Kompetenzentwicklung der Studierenden und die Entwicklung ihrer persönlichen Einsichten ab (Gerholz et al. 2015). Die Service-Ergebnisse sind je nach Projekt unterschiedlich.

\section{Design-Based-Research-Ansatz und Erhebungsdesign}

Die Einführung von Service-Learning wurde durch eine umfassende Begleitforschung flankiert, welche als Design-Based-Research-Ansatz konzipiert wurde. Design-Based-Research bezeichnet dabei ein methodologisches Paradigma, welches das Ziel verfolgt, innovative pädagogische Konzepte und Szenarien (bzw. Interventionen) in einer realen Anwendung systematisch zu erproben und aufbauend auf den 
Tab. 2 Übersicht Skalen (Wirksamkeit)

\begin{tabular}{|c|c|c|c|}
\hline Skala & Itemanzahl & Beispielitem (zu t1) & Cronbachs $\alpha$ \\
\hline $\begin{array}{l}\text { Selbstwirksamkeit (Schwarzer } \\
\text { und Jerusalem 1999) }\end{array}$ & 10 & $\begin{array}{l}\text { Auch bei überraschenden Ereig- } \\
\text { nissen glaube ich, daß ich gut mit } \\
\text { ihnen zurechtkommen werde }\end{array}$ & $\begin{array}{l}\mathrm{t} 1: 0,890 \\
\mathrm{t} 2: 0,907\end{array}$ \\
\hline $\begin{array}{l}\text { Engagementbereitschaft (Rein- } \\
\text { ders und Hildebrandt 2008) }\end{array}$ & 7 & $\begin{array}{l}\text { Ich würde einiges meiner Zeit } \\
\text { geben, um Menschen, die Unter- } \\
\text { stützung brauchen, zu helfen }\end{array}$ & $\begin{array}{l}\mathrm{t} 1: 0,752 \\
\mathrm{t} 2: 0,771\end{array}$ \\
\hline $\begin{array}{l}\text { Einstellung zum zivilgesellschaft- } \\
\text { lichen Engagement (Mabry 1998) }\end{array}$ & 5 & $\begin{array}{l}\text { Menschen haben die Verantwor- } \\
\text { tung zu helfen, um vorhandene } \\
\text { soziale Probleme zu lösen }\end{array}$ & $\begin{array}{l}\text { t1: } 0,847 \\
\mathrm{t} 2: 0,818\end{array}$ \\
\hline $\begin{array}{l}\text { Subjektiver Lernerfolg (Reinders } \\
\text { und Hildebrandt 2008; Ritzmann } \\
\text { et al. 2014) }\end{array}$ & 7 & $\begin{array}{l}\text { Ich könnte sicherlich einige Fra- } \\
\text { gen zu den Inhalten gut beantwor- } \\
\text { ten }\end{array}$ & $\begin{array}{l}t 1: 0,809 \\
t 2: 0,890\end{array}$ \\
\hline $\begin{array}{l}\text { Selbstbild (Reinders und Wittek } \\
\text { 2009) }\end{array}$ & 6 & $\begin{array}{l}\text { Durch die Lehrveranstaltung } \\
\text { werde ich mich selbst anders als } \\
\text { früher sehen }\end{array}$ & $\begin{array}{l}t 1: 0,854 \\
t 2: 0,868\end{array}$ \\
\hline
\end{tabular}

gewonnenen Ergebnissen sukzessive zu verbessern (Bell 2004). Interventionen werden dabei für diesen Beitrag als komplexe Problemlösungen in der Bildungspraxis (Plomp 2007) gesehen. Der Design-Based-Research-Ansatz soll Antworten darauf liefern, wie die Intervention Service-Learning gestaltet sein sollte, damit diese zur individuellen Förderung der Kompetenzentwicklung der Studierenden bestmöglich beitragen kann. Im konkreten bedeutet dies, wie die didaktische Modellierung (dargestellt an zentralen Gestaltungselementen) der Lehrveranstaltung Service-Learning aussehen soll.

Für die Umsetzung werden in einem ersten Schritt Interventionen entwickelt, welche die Merkmale des jeweiligen pädagogischen Konzepts beinhalten und dem Anwendungskontext durch kontinuierliche Verbesserung der Gestaltungselemente (dies kann als Designprozess beschrieben werden) der Intervention immer mehr gerecht werden (Reigeluth und Frick 1999). Der Design-Based-Research-Ansatz verfolgt damit zwei Ziele: Es sollen situationsspezifische Instrumente zur Verbesserung der Bildungspraxis entwickelt bzw. überprüft werden (praktischer Output), und gleichzeitig sollen kontextualisierte Theorien im Sinne von Theorien mittlerer Reichweite entstehen (theoretischer Output) (Edelson 2002; Reinmann 2005). Für den DesignBased Research-Ansatz ist charakteristisch, dass der Designprozess zwar grundlegend für den Forschungsprozess ist, von diesem aber abweichen kann. Dies liegt daran, dass sich die Intervention im Laufe des Designprozesses laufend verändert und der Forschungsprozess laufend adaptiert werden muss (so können sich beispielsweise auch die Erhebungsinstrumente während des Forschungsprozesses verändern, um gezielt auf eine Veränderung der Intervention reagieren zu können). Ein DesignBased-Research-Prozess ist damit vorausschauend und reflektierend: „Vorausschauend, weil Designs vor dem Hintergrund hypothetischer Lernprozesse und auf der Basis theoretischer Modelle implementiert und untersucht werden; reflektierend, weil Annahmen im Forschungsprozess analysiert und (mehrfach) überprüft werden“ (Reinmann 2005, S. 60). 
Die Implementierung der Service-Learning-Lehrveranstaltung in das Masterstudium Wirtschaftspädagogik verfolgte das Ziel, die Studierenden in für sie ungewohnte soziale Situationen zu bringen, in denen sie, mithilfe ihres fachlichen Wissens, selbsttätig Lösungen für Problemstellungen der jeweiligen Felder erstellen können. Die erfolgreiche Absolvierung dieser Anforderungen sollte, so die Vorannahme, Auswirkungen auf unterschiedlichen Ebenen der Persönlichkeitsentwicklung der Studierenden haben. Ein Ziel der begleitenden Studie war es daher, die Auswirkungen, die das Format auf die Persönlichkeitsentwicklung der Studierenden hat, nachzuzeichnen. Zu diesem Zweck wurden die in Tab. 2 dargestellten Konstrukte mittels standardisierter Skalen gemessen. Es wurde davon ausgegangen, dass sich durch die Beschäftigung mit sozial relevanten Themen und gesellschaftlich marginalisierten Menschen die Einstellung der Studierenden zu gesellschaftlichem Engagement verändern würde, was mittels der beiden Skalen ,Einstellung zum zivilgesellschaftlichen Engagement“ sowie „Engagementbereitschaft“ gemessen wurde. Daneben wurde davon ausgegangen, dass sich durch die eigenständige Bewältigung fachlicher Problemstellungen und die Erfahrung, dass die erarbeiteten Lösungen im sozialen Feld benötigt und angewandt werden, die Selbstwirksamkeitserwartung der Studierenden verändert.

Neben diesen drei direkt auf die Persönlichkeitsentwicklung abzielenden Konstrukten wurde auch erhoben, welche Erwartungen die Studierenden an die Lehrveranstaltung gehabt haben und inwiefern diese eingelöst wurden. Das Konstrukt subjektiver Lernerfolg misst den Abgleich zwischen den Erwartungen, die die Studierenden an ihren Lernerfolg durch das Modul haben, mit den tatsächlich gemachten Erfahrungen in Bezug auf ihren Kompetenzerwerb. Das Konstrukt Selbstbild misst den Abgleich der Erwartungen, die die Studierenden an das Modul hinsichtlich der Frage stellen, ob das Modul sie selbst, ihre Erwartungen, Handlungen und ihre Sicht auf sich selbst verändern wird, mit den im Modul dann gemachten Erfahrungen. Die innere Konsistenz der Skalen ist zu allen Erhebungszeitpunkten zufriedenstellend.

Die Erwartung an die Service-Learning-Veranstaltung war, dass die Studierenden am Ende einen Zuwachs in allen fünf Bereichen erzielen sollten. Dieser Zuwachs sollte in dieser spezifischen Lehrveranstaltung höher ausfallen, als er durch ähnliche Lehrveranstaltungen, in denen Studierende Lösungen für reale Unternehmen ohne Service-Gedanken finden müssen, erzielt werden könnte.

Das zweite Ziel, das durch die Begleitstudie verfolgt wurde, war, das Konzept selbst im Sinne einer umfassenden formativen Evaluation weiterzuentwickeln. Besonderes Augenmerk wurde dabei darauf gelegt, die zentralen Gestaltungselemente in der didaktischen Modellierung des Formats zu entdecken und in weiterer Folge zu optimieren.

\section{Methodik}

Als Untersuchungsdesign wurde ein komplexer Mixed-Method-Ansatz (Kuckartz 2014) gewählt, der aus einer standardisierten quasi-experimentellen Prä-Post-Erhebung und verschiedenen qualitativen Teilerhebungen besteht. 
Tab. 3 Quantitative Erhebung Übersicht

\begin{tabular}{llllll}
\hline & WS $14 / 15$ & SoSe 15 & WS $15 / 16$ & SoSe 16 & $\sum$ \\
\hline Experimentalgruppe & 18 & 22 & 16 & 26 & 82 \\
Kontrollgruppe & 29 & 19 & 28 & 29 & 105 \\
\hline
\end{tabular}

Das Design der quantitativen Untersuchung sah als Experimentalgruppe (EG) die Studierenden in den Service-Learning-Lehrveranstaltungen (Masterstudium Wirtschaftspädagogik) vor. Als Kontrollgruppe (KG) fungierte eine Lehrveranstaltung im Masterstudium Betriebswirtschaft (BW), in welcher die Studierenden ebenfalls Praxisprojekte aus dem Bereich Geschäftsmodelle und Standortpolitik in Kooperation mit realen Unternehmen abwickelten. Die Lehrveranstaltungsformate sind somit hinsichtlich der Dimension ,eigenständige Umsetzung von Projekten mit betriebswirtschaftlichem Inhalt und Praxiserfahrung" vergleichbar. Das Service-Learning-Konzept wurde über einen Zeitraum von vier Semestern, vom Wintersemester 2014/15 bis zum Sommersemester 2016, durchgeführt und weiterentwickelt. In jedem dieser Semester fand auch die vergleichbare Lehrveranstaltung im Master BW statt, die als Kontrollgruppe fungierte. In Summe wurden 82 Studierende in der Experimentalgruppe und 105 Studierende in der Kontrollgruppe befragt (Tab. 3). Bei einem systematischen Vergleich der Studierenden zeigte sich, dass es in der Experimentalgruppe einige deutlich ältere Studierende gibt. Dies ist dem Masterstudium Wirtschaftspädagogik geschuldet, das zur Lehre an Schulen befähigt. Personen, die bereits seit längerem im Arbeitsleben stehen, nutzen diese Option, um sich in Richtung Lehrberuf zu qualifizieren. Diese Personen sind aber gerade in Hinblick auf einige zentrale Kompetenzen, die durch das Service-Learning-Konzept gefördert werden sollen, nicht mit den anderen Studierenden vergleichbar, da sie bereits auf eine langjährige Praxiserfahrung zurückblicken. Daher wurden diese Personen $(N=7)$ aus den Berechnungen zum direkten Wirksamkeitsnachweis des Service-Learning-Konzeptes ausgeschlossen. Bei den Berechnungen zur Bewertung des Lehrveranstaltungskonzeptes wurden sie berücksichtigt. Abgesehen vom Alter gibt es keine signifikanten Unterschiede zwischen Kontroll- und Experimentalgruppe, weder was wichtige soziodemographische Aspekte noch was die Einstellungen zu sozialem Engagement vor Beginn der Lehrveranstaltung betrifft.

Neben der quantitativen Befragung wurden in jedem Semester auch qualitative Erhebungen durchgeführt. Die ausführlichste Erhebung wurde nach dem ersten Semester durchgeführt. Hierbei wurden alle im Wintersemester 2014/15 an der ServiceLearning-Lehrveranstaltung teilnehmenden Studierenden befragt. Die Studierenden arbeiteten in der Lehrveranstaltung in vier Gruppen zu jeweils vier bis fünf Personen zusammen. Mit diesen Kleingruppen wurden separate Gruppeninterviews mit einer Dauer von je ca. 90 min geführt. Die Durchführung der Interviews wurde, um sozial erwünschte bzw. positiv gefärbte Aussagen der Studierenden zu vermeiden, von einer den Studierenden unbekannten Person durchgeführt und transkribiert, welche auch keinerlei vorherige Verbindung zur Service-Learning-Lehrveranstaltung hatte. Neben den Gruppeninterviews mit den Studierenden wurden auch Interviews mit al- 
len KooperationspartnerInnen aus den Organisationen ${ }^{2}$ geführt. Alle Interviews waren als halbstrukturierte leitfadengestützte Interviews angelegt (Mayring 2002; Flick 2010) und die Auswertung der Daten erfolgte auf Basis der vollständig transkribierten Interviews mittels inhaltlich-strukturierender Inhaltsanalyse nach Kuckartz (2012).

Im Sinne des Design-Based Research-Ansatzes, der als iteratives Forschungsdesign angesehen werden kann, wurden die qualitativen Erhebungsformate in den folgenden Semestern den Veränderungen, die im didaktischen Design vorgenommen wurden, angeglichen. Die Erhebungen fokussierten von Semester zu Semester stärker auf diejenigen Bereiche, in denen Veränderungen vorgenommen wurden.

\section{Ergebnisse}

\subsection{Wirksamkeit des Service-Learning-Konzeptes}

Die in Tab. 4 dargestellten deskriptiven Kennwerte zeigen in allen erhobenen Konstrukten einen Zuwachs von Messzeitpunkt 1 zu Messzeitpunkt 2, der in der Experimentalgruppe stärker ausgeprägt ist als in der Kontrollgruppe.

Es wurden multivariate Varianzanalysen mit Messwiederholung (Field et al. 2012) berechnet, um signifikante Unterschiede sowohl zwischen den Messzeitpunkten als auch zwischen Experimental- und Kontrollgruppe feststellen zu können. Als Kontrollvariablen wurden Geschlecht, Alter, gesellschaftliches Engagement vor dem Seminar („Haben Sie sich bisher für einen guten Zweck oder für andere Menschen in irgendeiner Form engagiert?" Ausprägung ja/nein) sowie extrinsische Lernmotivation (3 Items, $\alpha=0,75$; Bsp.-Item: „Ich lerne und arbeite für das Modul, um möglichst gute Noten in den Prüfungen zu erzielen. ") und intrinsische Lernmotivation (3 Items, $\alpha=0,86$; Bsp.-Item: ,Ich lerne und arbeite im Modul, weil es mir Spaß macht. “) miteinbezogen. Das Gesamtmodell (MANOVA) zeigt einen signifikanten Interaktionseffekt zwischen der Variable Experimental- und Kontrollgruppe und dem Messzeitpunkt $(F=19.764, p=0,00)$. Bei Berücksichtigung der Kontrollvariablen zeigt sich weder ein Haupteffekt des Messzeitpunktes noch ein Haupteffekt der Variable Experimental- und Kontrollgruppe.

Die Vorannahme war, dass die Gruppe, die mit dem Service-Learning-Konzept konfrontiert ist, bis zum Ende der Lehrveranstaltung einen Zuwachs hinsichtlich ihrer Selbstwirksamkeit und ihrer Einstellung zu Engagement sowie ihrer Engagementbereitschaft erfährt, der stärker ausgeprägt ist als jener der Kontrollgruppe. Diese Hypothese wird durch die Interaktionseffekte, die für alle drei Konstrukte moderat ausfallen (Cohen 1992), bestätigt (siehe Tab. 5). Studierende, die die Lehrveranstaltung Service-Learning besucht haben, verzeichnen einen stärkeren Zuwachs

\footnotetext{
2 Nach der Erhebung im ersten Semester wurden in den folgenden drei Semestern lediglich die Perspektive der Studierenden in die Erhebung miteinbezogen, die Perspektive der Organisationen wurde ausgespart. Diese werden allerdings in einem Folgeprojekt im Wintersemester 2017/18 zu den Langzeitwirkungen der entwickelten Projekte befragt.
} 
Tab. 4 Deskriptive Kennwerte der Skalen (6stufige Likert-Skala, höhere Mittelwerte implizieren höhere Merkmalsausprägungen)

\begin{tabular}{|c|c|c|c|c|c|c|c|c|}
\hline & \multicolumn{4}{|l|}{$\mathrm{t} 1$} & \multicolumn{4}{|l|}{$\mathrm{t} 2$} \\
\hline & \multicolumn{2}{|c|}{ Experimentalgruppe } & \multicolumn{2}{|c|}{ Kontrollgruppe } & \multicolumn{2}{|c|}{ Experimentalgruppe } & \multicolumn{2}{|c|}{ Kontrollgruppe } \\
\hline & MW & SD & MW & SD & MW & SD & MW & SD \\
\hline Selbstwirksamkeit & 4,50 & 0,57 & 4,45 & 0,77 & 4,88 & 0,54 & 4,46 & 0,75 \\
\hline $\begin{array}{l}\text { Einstellung } \\
\text { Engagement }\end{array}$ & 4,64 & 0,91 & 4,76 & 0,78 & 5,02 & 0,71 & 4,65 & 0,76 \\
\hline $\begin{array}{l}\text { Engagement- } \\
\text { bereitschaft }\end{array}$ & 4,41 & 0,63 & 4,61 & 0,76 & 4,83 & 0,61 & 4,45 & 0,67 \\
\hline
\end{tabular}

Tab. 5 Interaktionseffekte

\begin{tabular}{llll}
\hline & F-Wert & $P$-Wert & Partielles Eta-Quadrat \\
\hline Selbstwirksamkeit & 27.549 & 0,00 & 0,142 \\
Einstellung zu zivilgesellschaftlichen Engagement & 31.362 & 0,00 & 0,132 \\
Soziale Engagementbereitschaft & 25.460 & 0,00 & 0,158 \\
\hline
\end{tabular}

an Selbstwirksamkeit, sie schätzen gesellschaftliches Engagement als wichtiger ein und ihre Engagementbereitschaft erhöht sich im Vergleich zur Kontrollgruppe.

Neben dem Einfluss, den das Service-Learning-Konzept auf die Persönlichkeitsentwicklung der Studierenden zu nehmen vermag, war es in der quantitativen Evaluation im Hinblick auf die Weiterentwicklung des Konzeptes wichtig zu prüfen, welche Effekte die Studierenden der Lehrveranstaltung zuschreiben. Dazu wurden mittels multivariater Varianzanalysen mit Messwiederholung geprüft, ob sich die beiden Konstrukte Selbstbild und Subjektiver Lernerfolg aufgrund des Seminars ändern. Als Kontrollvariablen wurden wiederum Geschlecht, Alter, gesellschaftliches Engagement vor dem Seminar sowie extrinsische und intrinsische Lernmotivation miteinbezogen (vgl. Tab. 6). Im Gesamtmodell (MANOVA) zeigt sich ein signifikanter Haupteffekt der Experimental- und Kontrollgruppe $(F=47.955 ; p=0,00)$ sowie ein signifikanter Interaktionseffekt zwischen Experimental- und Kontrollgruppe und dem Messzeitpunkt $(F=18.174 ; p=0,00)$.

Bei näherer Betrachtung der Effekte (Tab. $7 \mathrm{zu}$ den deskriptiven Kennwerten) wird deutlich, dass sowohl bei den Erwartungen an die Veränderung des Selbstbildes als auch bei den Erwartungen an den Lernerfolg durch das Modul die Experimentalgruppe über der Kontrollgruppe lag. Der Zuwachs im Semester war in der Experimentalgruppe vor allem hinsichtlich der Veränderung des Selbstbildes stark ausgeprägt, während die Kontrollgruppe ungefähr auf dem Ausgangsniveau verblieb. Dies könnte so interpretiert werden, dass die Erwartungen der Kontrollgruppe durch das Seminar erfüllt wurden, während die Erwartungen der Experimentalgruppe durch das Service-Learning-Konzept übertroffen wurden.

Die Ergebnisse aus den qualitativen Erhebungen ermöglichen einen Einblick in die Erfahrungen, die die Studierenden während der Service-Learning-Veranstaltung gemacht haben. Sie beziehen sich dabei häufig auf ähnliche Ebenen, die im quantitativen Teil mittels der berichteten Skalen abgefragt wurden, womit sich die gemessenen Veränderungen aus subjektiver Sicht der Studierenden beschreiben lassen. 
Tab. 6 Haupt- und Interaktionseffekt

\begin{tabular}{lccc}
\hline & F-Wert & $P$-Wert & Partielles Eta-Quadrat \\
\hline Haupteffekt & & & \\
Experimental- und Kontrollgruppe & & & \\
Subjektiver Lernerfolg & 31.913 & 0,00 & 0,161 \\
Veränderung Selbstbild & 73.306 & 0,00 & 0,306 \\
Interaktionseffekt & & & \\
Subjektiver Lernerfolg & 20.525 & 0,00 & 0,110 \\
Veränderung Selbstbild & 25.162 & 0,00 & 0,132 \\
\hline
\end{tabular}

Tab. 7 Deskriptive Kennwerte der Skalen (6stufige Likert-Skala, höhere Mittelwerte implizieren höhere Merkmalsausprägungen)

\begin{tabular}{|c|c|c|c|c|c|c|c|c|}
\hline & \multicolumn{4}{|l|}{$\mathrm{t} 1$} & \multicolumn{4}{|l|}{$\mathrm{t} 2$} \\
\hline & \multicolumn{2}{|c|}{ Experimentalgruppe } & \multicolumn{2}{|c|}{ Kontrollgruppe } & \multicolumn{2}{|c|}{ Experimentalgruppe } & \multicolumn{2}{|c|}{ Kontrollgruppe } \\
\hline & MW & SD & MW & SD & MW & $\mathrm{SD}$ & MW & $\mathrm{SD}$ \\
\hline $\begin{array}{l}\text { Subjektiver } \\
\text { Lernerfolg }\end{array}$ & 4,67 & 0,55 & 4,36 & 0,72 & 5,26 & 0,57 & 4,45 & 0,78 \\
\hline Selbstbild & 3,60 & 0,94 & 3,16 & 0,91 & 4,26 & 0,73 & 3,09 & 0,88 \\
\hline
\end{tabular}

Der Nutzen für die Studierenden liegt aus Sicht der Organisationen im Einblick, den sie in andere soziale Schichten und Lebensformen erhalten. Die Studierenden selbst verweisen darauf, dass Einblick in eine soziale Organisation sowie die Tatsache, einmal mit Armut im Land konfrontiert zu werden, besonders sinnvoll sei. Die im Konzept Service-Learning zentrale Sensibilisierung für soziale Aspekte der Gesellschaft scheint erreicht worden zu sein. So stellt z. B. ein/e Studierende/r fest, „man schämt sich irgendwie im Nachhinein ein bisschen fast für seine Berührungsängste, seine Sachen, die man sich vorher gedacht hat [...]“. Die Studierenden sprechen in diesem Zusammenhang davon, dass ihnen die eigene privilegierte Stellung wieder zu Bewusstsein gekommen sei. Hier meinen die Studierenden auch, dass wirtschaftliche Aspekte zugunsten von sozialen Aspekten in den Hintergrund gerückt wären. „Also ich habe eher (.) eher sozial am meisten gelernt (.), eben da diesen Einblick zu bekommen in die andere Seite, dass dass dass es nicht nur wirtschaftliche Sichtweisen gibt, sondern auch solche sozialen Komponenten." Angestoßen werden diese Reflexionen durch die persönliche Betroffenheit, die das Projekt auslöst. Einige Studierendengruppen arbeiten auch über die Lehrveranstaltung hinaus mit den Organisationen zusammen, andere beginnen sich privat zu engagieren , [.] ich glaube ich bin mein Leben lang jetzt verbunden" mit der Organisation. Wieder andere berührt das Zusammentreffen so, dass sie in ihrem privaten Umfeld viel von den Organisationen erzählen: ,die ganze Familie weiß jetzt [...] “, was diese Organisation ist. Dies gibt einen Hinweis darauf, dass die Studierenden auch nach dem Besuch der Lehrveranstaltung gesellschaftlich engagiert sind.

Von großer Bedeutsamkeit scheinen eine gute Einbindung in die Organisation und vor allem der persönliche Kontakt zu den KlientInnen zu sein. Jene Studierenden, die diesen Einblick erhielten, berichten darüber, dass die Arbeit für die Organisationen und ihre KlientInnen für sie persönlich sehr wichtig geworden wäre. Sie hätten 
Tab. 8 Designvariablen

\begin{tabular}{llll}
\hline Skala & Itemanzahl & Beispielitem & Cronbachs $\alpha$ \\
\hline $\begin{array}{l}\text { Anwendungsbezug (Ritzmann } \\
\text { et al. 2014) }\end{array}$ & 5 & $\begin{array}{l}\text { In der LV hatte ich die Möglich- } \\
\text { keit, Dinge, die ich in zukünttigen } \\
\text { Berufsfeldern umsetzen soll, } \\
\text { schon einmal auszuprobieren }\end{array}$ & 0,813 \\
$\begin{array}{l}\text { Problembasiertes Lernen (Ritz- } \\
\text { mann et al. 2014) }\end{array}$ & 5 & $\begin{array}{l}\text { Es wurden zuerst Probleme the- } \\
\text { matisiert und durch ihre Bearbei- } \\
\text { tung habe ich die Inhalte gelernt } \\
\text { Das Lernen hat Spaß gemacht }\end{array}$ & 0,789 \\
$\begin{array}{l}\text { Subjektive Freude (Ritzmann } \\
\text { et al. 2014) }\end{array}$ & 3 & $\begin{array}{l}\text { Ich hatte Gelegenheit das Gelern- } \\
\text { te zu reflektieren }\end{array}$ & 0,764 \\
$\begin{array}{l}\text { Integration des Gelernten (Ritz- } \\
\text { mann et al. 2014) }\end{array}$ & 5 & & \\
\hline
\end{tabular}

erfahren, dass sie „etwas Echtes machen“ und „etwas Echtes bewegen “ könnten, das nicht nur Auswirkungen auf die Noten im Studium hätte, sondern tatsächlich das Leben anderer positiv beeinflussen könnte. ,, [...] echt die [KlientInnen] haben sich so gefreut, ich habe das gar nicht glauben können“. Diese Komponenten könnten dazu beitragen, dass die Selbstwirksamkeitserwartung der Studierenden gestärkt wird, da sie die Erfahrung machen, dazu fähig zu sein, in einem für sie zunächst fremden Umfeld wirksame Interventionen zu setzen.

\subsection{Bewertung des didaktischen Designs}

Neben der Überprüfung der Wirksamkeit des Service-Learning-Formates war es von Interesse, ob die didaktische Gestaltung im Allgemeinen zufriedenstellend angelegt war und wichtige Ziele des Formates wie Praxisbezug erreicht werden konnten. Daher wurden nach Ende der Lehrveranstaltung die Variablen Anwendungsbezug, Problembasiertes Lernen, Subjektive Freude und Integration des Gelernten erhoben (Tab. 8), die eine Bewertung des didaktischen Designs ermöglichen.

In multivariaten Varianzanalysen zeigte sich bei Kontrolle des Geschlechts, des Alters sowie der intrinsischen und extrinsischen Lernmotivation für das Gesamtmodell ein signifikanter Unterschied zwischen Experimental- und Kontrollgruppe $(F=7862, p=0,00)$. Bei Betrachtung der einzelnen Designvariablen finden sich signifikante Unterschiede für die Bereiche Problembasiertes Lernen $(F=12.540$, $p=0,00$, et $\left.a^{2}=0,070\right)$, Anwendungsbezug $\left(F=15.077, p=0,00\right.$, eta $\left.{ }^{2}=0,083\right)$ sowie Integration des Gelernten $\left(F=23.887, p=0,00\right.$, eta $\left.^{2}=0,125\right)$.

Studierende, die die Lehrveranstaltung Service-Learning besuchten, bewerteten das didaktische Design besser, als die Kontrollgruppe ihre Lehrveranstaltung bewertete, wobei der Unterschied bei der Integration des Gelernten am stärksten ausfiel. Generell sind die Effekte eher schwach ausgeprägt, was auch Tab. 9 zeigt. Hinsichtlich der subjektiven Freude gab es keinen signifikanten Unterschied. 
Tab. 9 Deskriptive Kennwerte der Skalen (6stufige Likert-Skala, höhere Mittelwerte implizieren höhere Merkmalsausprägungen)

\begin{tabular}{lllll}
\hline & \multicolumn{2}{l}{ Experimentalgruppe } & \multicolumn{2}{l}{ Kontrollgruppe } \\
& MW & SD & MW & SD \\
\hline Anwendungsbezug & 4,64 & 0,84 & 4,03 & 0,86 \\
Problembasiertes Lernen & 4,93 & 0,81 & 4,32 & 0,89 \\
Subjektive Freude & 5,16 & 0,82 & 4,91 & 0,93 \\
Integration des Gelernten & 4,87 & 0,69 & 4,22 & 0,84 \\
\hline
\end{tabular}

\subsection{Didaktische Adaptionen in den unterschiedlichen Semestern und die Reaktionen der Studierenden}

Fall 1 (WiSe 2014/15). Die Service-Learning-Veranstaltung wurde im Wintersemester 2014/15 das erste Mal durchgeführt. Vor Beginn der Lehrveranstaltung skizzierten die LehrveranstaltungsleiterInnen in Zusammenarbeit mit den sozialen Organisationen den Projektauftrag bzw. -ablauf. Die Inhalte der studentischen Projekte sowie deren Umsetzung wurden somit in groben Zügen bereits vor Lehrveranstaltungsbeginn festgehalten. Weiters wurden die konkreten AnsprechpartnerInnen innerhalb der Organisationen für die Studierenden festgelegt.

Die Ergebnisse der empirischen Erhebungen am Ende des Semesters zeigten grundsätzlich eine sehr positive Resonanz der Studierenden auf das Lehr-Lernformat Service-Learning. Unsicherheiten zeigten sich hinsichtlich des Umstandes, dass sich der Fokus einiger Projekte während des Semesters geändert hatte, da die ursprüngliche Zielsetzung sich als uneinlösbar herausgestellt hatte. Zudem merkten einige Studierenden an, es sei für sie belastend gewesen, dass von Seiten der sozialen Organisationen teilweise andere Anforderungen und Wünsche an sie gestellt wurden als von Seiten der Lehrenden. Kritisch wurde von den Studierenden die Benotung der Kursleistung gesehen, da sie kein klares Schema für die Benotung erkennen konnten. Die ergibt sich aus dem Umstand, dass sich die Projekte und die damit verbundenen Anforderungen im Laufe des Semesters immer (wieder) verändern können. Für die einzelnen Problemstellungen kann es somit keine vorgefertigte Musterlösung geben.

Fall 2 (SoSe 2015). Im Sommersemester 2015 wurde das generelle Design der Lehrveranstaltung (acht Lehrveranstaltungstermine, Pflichtfach) beibehalten. Beim ersten Lehrveranstaltungstermin wurden aber vier Punkte zusätzlich thematisiert, welche sich aus den Ergebnissen der ersten Interviewstudie ergaben. Erstens wurde mit den Studierenden sehr offen darüber diskutiert, dass diese sowohl den Ansprüchen der Organisationen als auch den Ansprüchen der Lehrveranstaltung genügen müssten, was bedeutet, dass sie zwei unterschiedliche AuftraggeberInnen hätten. Die Studierenden wurden aufgefordert, mit diesem Spannungsverhältnis produktiv umzugehen und vor allem ihre eigene Meinung stärker einzubringen. Zweitens wurde die Ergebnisoffenheit der Projekte angesprochen, d. h. der Umstand, dass sich diese, trotz der Abstimmung mit den Organisationen, im Laufe des Semesters ändern könnten. Drittens wurde bereits im Vorfeld mit den Organisationen besprochen, dass die 
Studierenden möglichst viel Kontakt mit den jeweiligen KlientInnen, d.h. mit den Personen, für die die jeweilige Organisation zuständig ist, bekämen. Viertens wurde das Thema der Benotung mit den Studierenden besprochen und darauf hingewiesen, dass es sich um offene Projekte handelte, bei denen es keine Musterlösung und auch keine exakten Prozentwerte für das Erreichen einer bestimmten Note geben könnte.

Die Gruppendiskussionen nach Ende des Semesters zeigten, dass die Thematisierung der unterschiedlichen Ansprüche seitens der sozialen Organisationen und der Lehrenden sowie der Ergebnisoffenheit der Projekte sehr positiv aufgenommen wurde. Die Studierenden meinten, dass eben diese Situationen in ihrem späteren Arbeitsalltag auch vorkommen würden und sie diese daher als Lernchance begriffen hätten. Hier wurde vor allem der Umstand hervorgehoben, dass dies den Studierenden ermöglichte, sich selbst stärker in die Gestaltung der Projekte einbringen zu können, als dies in herkömmlichen Seminaren der Fall wäre. Der Kontakt mit den KlientInnen wurde von den Studierenden als sehr positiv empfunden, auch wenn es nicht bei allen Projektgruppen möglich war, diesen intensiv zu erreichen. Der in dem ersten Fall stark kritisierte Punkt der Benotung spielte im zweiten Fall keine große Rolle mehr. Die Studierenden konnten mit dem Wissen, dass es sich um eine teiloffene Leistungsanforderung bzw. -beurteilung handeln würde, gut umgehen.

Fall 3 (WiSe 2015/16). Im Wintersemester 2015/16 wurden (neben den Adaptionen von Fall 2) wesentliche Veränderungen hinsichtlich der Abstimmung mit den sozialen Organisationen im Vorfeld bzw. während der Lehrveranstaltung vorgenommen. Die Projektsteuerung wurde stärker in die Hände der Organisationen gelegt. So wurde die genaue Problemstellung nicht im Vorfeld mit den Organisationen abgestimmt. Dies bot den Vorteil, dass den Studierenden damit die Herausforderung geboten wurde, das Problem erst zu identifizieren und selbstständig, in Abstimmung mit den Organisationen, eine Lösungsstrategie zu entwickeln. Den Studierenden wurde diesbezüglich verdeutlicht, dass sie mit den Organisationen einen ebenbürtigen Diskurs als fachliche Experten führen könnten und sollten. Die Organisationen wurden im Vorfeld der Lehrveranstaltung nochmals explizit gebeten, den Studierenden den Kontakt mit ihren KlientInnen zu ermöglichen. Auch die Studierenden wurden in der Lehrveranstaltung beauftragt, diesen Kontakt aktiv zu suchen. Die Lehrenden versuchten, während der Lehrveranstaltung die Rolle eines Coaches einzunehmen und nicht länger die inhaltliche Steuerung zu übernehmen. Dies führte zu einer stärkeren Einzelbetreuung der Kleingruppen. Die acht Lehrveranstaltungstermine wurden damit zwar als Struktur beibehalten; während des Semesters wurden diese aber, in Abhängigkeit vom Fortschritt der Studierenden, teilweise in Coachingtermine für einzelne Gruppen umgewandelt.

Die offene Herangehensweise an die Problemstellung wurde in den Gruppeninterviews nach Ende des Semesters als sehr positiv beurteilt. Es wurde angemerkt, dass dies zwar eine große Herausforderung darstellte, damit aber auch ein besseres Arbeiten und Lernen in und mit den Projekten ermöglicht wurde. Die Studierenden äußerten dabei auch den Wunsch, das selbstständige Entscheiden in den Projekten beizubehalten. Die flexible Handhabung der Lehrveranstaltungstermine während des Semesters, die in Abstimmung mit dem Fortschritt der Gruppen vorgenommen wurde, wurde ebenfalls positiv bewertet. Weiters wurde angemerkt, dass die Beurteilung 
insgesamt als fair und darüber hinaus die Beurteilungen in diesem Seminar eher als nebensächlich empfunden wurden.

Fall 4 (SoSe 2016). Im Sommersemester 2016 wurden die bisher dargestellten Gestaltungsentscheidungen direkt übernommen. Die Studierenden wurden am Beginn noch gebeten, weder die Ideen bzw. Vorgaben der Organisationen noch die der Lehrenden einfach zu übernehmen, sondern immer kritisch dazu Stellung zu nehmen. Dieses Semester stellt somit den Endpunkt des Design-Zyklus dar.

Die Ergebnisse der Befragungen nach Ende des Semesters ließen ein gespaltenes Bild entstehen. Die Gruppendiskussion zeigte, dass die Studierenden mit der Gestaltung der Lehrveranstaltung sehr zufrieden waren. Hier ergaben sich keine großen Veränderungswünsche bzw. -potenziale. Es zeigte sich aber, dass es in diesem Semester nicht gelungen war, die Studierenden mit den KlientInnen der sozialen Organisationen in Kontakt zu bringen. Dies lag zum einen an manchen der Problemstellungen, die keinen direkten KlientInnenkontakt erforderlich machten. Zum anderen wurde der Kontakt aber auch nicht von den Organisationen forciert, da diese befürchteten, ihre KlientInnen könnten dies als aufdringlich empfinden.

\section{Identifizierte Gestaltungselemente von Service-Learning}

Im Verlauf der forschenden Begleitung der Lehrveranstaltung (LV) mittels des Design-Based-Research-Ansatzes wurden fünf Gestaltungselemente identifiziert, die maßgeblich zum Gelingen oder Misslingen des Service-Learning-Konzeptes beitragen (siehe Tab. 10).

Wesentlich für die Wirkung des Formats Service-Learning erscheinen zusammengefasst zwei Punkte. Erstens dass ein Service-Prozess die Studierenden vor eine offene Problemsituation mit ihrer eigenen Logik und den damit verbundenen Herausforderungen stellen soll. Die Auseinandersetzung mit dem Finden der Problemstellung und vor allem das Handeln unter Unsicherheit ist dabei ein wesentlicher Aspekt. Dieser wird nicht für die Studierenden im Vorfeld der Lehrveranstaltung geklärt, sondern stellt einen wichtigen Teil des Lern- bzw. Serviceprozesses dar. Zweitens muss eine Integration der Studierenden in die sozialen Organisationen insbesondere hinsichtlich eines Kontakts mit den KlientInnen dieser Organisationen gelingen. Dies scheint einen wesentlichen Einfluss auf die Wirksamkeit von ServiceLearning zu haben, da damit die Projekte nicht für die eigene Note, die Lehrenden, die Universität oder die sozialen Organisationen, sondern für konkrete Personen, welche sich in einer sozialen Notlage befinden, bearbeitet werden.

Von Interesse im Sinne des Design-Based-Research-Ansatzes war, ob sich die Veränderungen, die im didaktischen Design von Semester zu Semester vorgenommen wurden, in der Wirksamkeit der Service-Learning-Lehrveranstaltung wiederspiegeln würden. Zu diesem Zweck wurde für die Variablen Selbstwirksamkeit, Einstellung zu zivilgesellschaftlichem Engagement, Soziale Engagementbereitschaft, Selbstbild und Subjektiver Lernerfolg die Differenz der Post- und der Prämessung ermittelt; danach wurden Varianzanalysen mit dem Differenzbetrag als abhängiger Variable und den unterschiedlichen Semestern durchgeführt. Veränderungen des di- 
Tab. 10 Veränderung der Gestaltungselemente über die vier Semester

\begin{tabular}{|c|c|c|c|c|}
\hline & Fall 1 & Fall 2 & Fall 3 & Fall 4 \\
\hline $\begin{array}{l}\text { Verhältnis der } \\
\text { Steuerung des } \\
\text { Projekts durch Leh- } \\
\text { rende bzw. soziale } \\
\text { Organisationen }\end{array}$ & $\begin{array}{l}\text { Absprache mit } \\
\text { den Organisa- } \\
\text { tionen vor LV- } \\
\text { Beginn über die } \\
\text { genaue Problem- } \\
\text { stellung }\end{array}$ & $\begin{array}{l}\text { Beim ersten LV-Ter- } \\
\text { min wurde mit den } \\
\text { Studierenden klar be- } \\
\text { sprochen, dass diese } \\
\text { zwei Ansprechpartner- } \\
\text { Innen haben werden }\end{array}$ & $\begin{array}{l}\text { Projektsteuerung } \\
\text { wurde stärker } \\
\text { an die Organisa- } \\
\text { tionen gegeben } \\
\text { (Handeln unter } \\
\text { Unsicherheit) }\end{array}$ & $\begin{array}{l}\text { Wie } \\
\text { Fall } 3\end{array}$ \\
\hline $\begin{array}{l}\text { Handeln unter } \\
\text { Unsicherheit }\end{array}$ & $\begin{array}{l}\text { Absprache mit } \\
\text { den Organisa- } \\
\text { tionen vor LV- } \\
\text { Beginn über die } \\
\text { genaue Problem- } \\
\text { stellung }\end{array}$ & Wie Fall 1 & $\begin{array}{l}\text { Problemstellung } \\
\text { wurde bewusst } \\
\text { nicht mit den } \\
\text { Organisationen } \\
\text { geklärt (nur Pro- } \\
\text { blembereich) }\end{array}$ & $\begin{array}{l}\text { Wie } \\
\text { Fall } 3\end{array}$ \\
\hline $\begin{array}{l}\text { Ausmaß der In- } \\
\text { tegration in die } \\
\text { Organisation }\end{array}$ & $\begin{array}{l}\text { Kontakt mit der } \\
\text { Organisation } \\
\text { eingefordert }\end{array}$ & $\begin{array}{l}\text { Kontakt mit den Kli- } \\
\text { entInnen eingefordert }\end{array}$ & Wie Fall 2 & $\begin{array}{l}\text { Wie } \\
\text { Fall } 2\end{array}$ \\
\hline $\begin{array}{l}\text { Individuelles Coa- } \\
\text { ching/Beratung }\end{array}$ & $\begin{array}{l}\text { Acht LV-Ein- } \\
\text { heiten für die } \\
\text { gesamte Gruppe }\end{array}$ & Wie Fall 1 & $\begin{array}{l}\text { Mehr Einzel- } \\
\text { termine je nach } \\
\text { Bedarf (bessere } \\
\text { Projektsteuerung) }\end{array}$ & $\begin{array}{l}\text { Wie } \\
\text { Fall } 3\end{array}$ \\
\hline Beurteilung & $\begin{array}{l}\text { Beurteilt wurde } \\
\text { Projektbericht } \\
\text { und -ergebnis }\end{array}$ & $\begin{array}{l}\text { Benotung wurde in } \\
\text { der Lehrveranstaltung } \\
\text { dezidiert thematisiert }\end{array}$ & $\begin{array}{l}\text { Anforderungen } \\
\text { an den Projektbe- } \\
\text { richt wurden klar } \\
\text { herausgestrichen }\end{array}$ & $\begin{array}{l}\text { Wie } \\
\text { Fall } 3\end{array}$ \\
\hline
\end{tabular}

Tab. 11 Deskriptive Veränderung in den Zuwächsen über die Semester

\begin{tabular}{|c|c|c|c|c|c|c|c|c|c|c|}
\hline & \multicolumn{2}{|c|}{$\begin{array}{l}\text { Selbstwirksam- } \\
\text { keit }\end{array}$} & \multicolumn{2}{|c|}{$\begin{array}{l}\text { Einstellung } \\
\text { Engagement }\end{array}$} & \multicolumn{2}{|c|}{$\begin{array}{l}\text { Engagement- } \\
\text { bereitschaft }\end{array}$} & \multicolumn{2}{|c|}{$\begin{array}{l}\text { Subjektiver } \\
\text { Lernerfolg }\end{array}$} & \multicolumn{2}{|c|}{ Selbstbild } \\
\hline & MW & SD & MW & SD & MW & SD & MW & SD & MW & SD \\
\hline $\begin{array}{l}\text { WS } \\
14 / 15\end{array}$ & 0,16 & 0,51 & 0,31 & 0,83 & 0,18 & 0,80 & 0,50 & 0,70 & 0,42 & 1,13 \\
\hline SS 15 & 0,45 & 0,33 & 0,53 & 0,90 & 0,46 & 0,50 & 0,75 & 0,58 & 0,29 & 0,74 \\
\hline $\begin{array}{l}\text { WS } \\
15 / 16\end{array}$ & 0,56 & 0,31 & 0,34 & 0,59 & 0,46 & 0,54 & 0,56 & 0,36 & 0,73 & 0,86 \\
\hline$\underline{\text { SS } 16}$ & 0,36 & 0,43 & 0,35 & 0,69 & 0,52 & 0,47 & 0,55 & 0,50 & 1,11 & 0,82 \\
\hline
\end{tabular}

daktischen Designs sollten sich bestenfalls in einer Zunahme der Differenz zwischen Post- und Prämessung abbilden. Tab. 11 zeigt die deskriptiven Befunde der Veränderungen in den Zuwächsen über die Semester.

Es zeigten sich signifikante Effekte für eine Veränderung des Zuwachses an Selbstwirksamkeitserwartung $(\mathrm{F}=3,083 ; p=0,032)$ sowie eine Veränderung des Selbstbildes $(\mathrm{F}=4,014 ; p=0,010)$ zwischen den Semestern.

Bei der Veränderung des Selbstbildes zeigt sich ein leichter Abfall im SoSe 2015 und darauf folgend eine Steigerung in den beiden nachfolgenden Semestern. Post Hoc-Tests ergaben, dass es keinen signifikanten Unterschied zwischen dem WiSe 2014/15 und dem SoSe 2015 gibt, diese beiden Semester sich jedoch zu den nachfolgenden unterscheiden. Bezüglich der Selbstwirksamkeitserwartung zeigte sich zunächst ein Anstieg mit jedem Semester bis zum WiSe 2015/16. Im SoSe 2016 
Tab. 12 Deskriptive Kennwerte der Designvariablen (6stufige Likert-Skala, höhere Mittelwerte implizieren höhere Merkmalsausprägungen)

\begin{tabular}{lllllllll}
\hline & \multicolumn{2}{c}{ Anwendungsbezug } & \multicolumn{2}{l}{$\begin{array}{l}\text { Problembasiertes } \\
\text { Lernen }\end{array}$} & \multicolumn{3}{l}{$\begin{array}{l}\text { Subjektive } \\
\text { Freude }\end{array}$} & \multicolumn{3}{l}{$\begin{array}{l}\text { Integration des } \\
\text { Gelernten }\end{array}$} \\
& MW & SD & MW & SD & MW & SD & MW & SD \\
\hline WS & 4,58 & 0,78 & 5,00 & 0,61 & 4,96 & 1,24 & 4,70 & 0,83 \\
$14 / 15$ & & & & & & & & \\
SS 15 & 4,56 & 0,84 & 5,04 & 0,79 & 5,20 & 0,67 & 4,65 & 0,65 \\
WS & 5,16 & 0,41 & 5,28 & 0,52 & 5,60 & 0,43 & 5,39 & 0,42 \\
$15 / 16$ & & & & & & & & \\
SS 16 & 4,42 & 0,96 & 4,58 & 0,99 & 5,00 & 0,67 & 4,85 & 0,61 \\
\hline
\end{tabular}

fiel der Zuwachs an Selbstwirksamkeitserwartung jedoch wieder ab. Post Hoc-Tests zeigen, dass die signifikant unterschiedlichen Gruppen die des WiSe 2014/15 und des WiSe 2015/16 sind. Im WiSe 2014/15 erfuhren die Studierenden den geringsten Zuwachs an Selbstwirksamkeit, im WiSe 2015/16 den stärksten.

Bezüglich der Designvariablen wurden der Anwendungsbezug $(\mathrm{F}=3,000$; $p=0,036)$ und die Integration des Gelernten $(\mathrm{F}=4,673 ; p=0,005)$ zwischen den Semestern signifikant unterschiedlich eingeschätzt. Wie in Tab. 12 dargestellt, zeigte sich, dass diese in den ersten beiden Semestern (WiSe 2014/15 und SoSe 2015) ähnlich gut eingeschätzt wurden, im WiSe 2015/16 werden sie deutlich höher eingeschätzt, im SoSe 2016 sinkt die Einschätzung wiederum.

Sowohl bei den Designvariablen als auch beim Zuwachs an Selbstwirksamkeitserwartung gab es somit im SoSe 2016 einen Abfall. In diesem Semester gaben die Studierenden in der qualitativen Befragung an, dass sie zwar einen guten Kontakt zu den Organisationen etablieren konnten, jedoch wenig Kontakt zu den KlientInnen hatten. Gerade der direkte Kontakt zu den KlientInnen wurde von denjenigen Studierendengruppen der anderen Semester, die stabile Kontakte aufbauen konnten, als besonders wertvoll beschrieben, da erstens Einblicke in fremde Lebenswelten gewonnen werden konnten und zweitens im unmittelbaren Kontakt die Erfahrung gemacht wurde, dass die Maßnahmen, die von den Studierenden entwickelt und tatsächlich umgesetzt wurden, einen Einfluss auf das Leben anderer hätte. Es steht zu vermuten, dass das Fehlen des direkten Kontaktes zu den KlientInnen zu einer geringeren Wirksamkeit und einer schlechteren Bewertung der didaktischen Modellierung des Service-Learning-Formates führt.

Die Studie unterliegt in ihrer Aussagekraft Grenzen, die sich sowohl aus der Gestaltung der Lehrveranstaltung als auch aus dem empirischen Design ergeben. So wurde die Lehrveranstaltung nur an einer Universität durchgeführt und hier nur für einen Studiengang. Daraus ergibt sich auch für die Begleitforschung eine geringe Fallanzahl und damit einhergehende Restriktionen für mathematische Modelle. Auch die Veränderung der qualitativen Erhebungsformate im Laufe der Semester macht einen direkten Vergleich der Semester nicht möglich. Weiters fokussierte die vorgestellte Untersuchung vor allem auf den Einfluss den Service-Learning auf die Persönlichkeitsentwicklung hat. Die fachliche Kompetenzentwicklung wurde nicht überprüft. In Zukunft wäre es daher von Nöten zu prüfen, welchen Einfluss Service- 
Learning auf Studierende verschiedener Fachrichtungen nimmt und wie sich die Kompetenzentwicklung im fachlichen Bereich ausgestaltet.

Funding Open access funding provided by University of Graz.

Open Access Dieser Artikel wird unter der Creative Commons Namensnennung 4.0 International Lizenz (http://creativecommons.org/licenses/by/4.0/deed.de) veröffentlicht, welche die Nutzung, Vervielfältigung, Bearbeitung, Verbreitung und Wiedergabe in jeglichem Medium und Format erlaubt, sofern Sie den/die ursprünglichen Autor(en) und die Quelle ordnungsgemäß nennen, einen Link zur Creative Commons Lizenz beifügen und angeben, ob Änderungen vorgenommen wurden.

\section{Literatur}

Arney, J. (2006). Uniting community and university through service learning. Business Communication Quarterly, 69, 195-198.

Astin, A. W., Vogelgesang, L. J., Ikeda, E. K., \& Yee, J. A. (2000). How service-learning affects students. Oakland: University of California.

Backhaus-Maul, H., \& Roth, C. (2013). Service-Learning an Hochschulen in Deutschland. Wiesbaden: Springer.

Bartsch, G. (2009). Do it! Experiences with service-learning. In M. Moore \& C. Lan (Hrsg.), Servicelearning in higher education (S. 329-336). Indianapolis: University of Indianapolis Press.

Bell, P. (2004). On the theoretical breadth of design-based research in education. Educational Psychologist, $39(4), 243-253$.

Boss, J. (1994). The effect of community service work on the moral development of college ethics students. Journal of Moral Education, 23, 183-198.

Bringle, R.G., \& Clayton, P.H. (2012). Civic education through service-learning. In L. McIlrath, A. Lyons \& R. Munck (Hrsg.), Higher education and civic engagement: comparative perspectives (S. 101-123). New York: Palgrave Macmillan.

Buschfeld, D. (2003). Draußen vom Lernfeld komm' ich her..? Ein Plädoyer für einen alläglichen Umgang mit Lernsituationen (S. 1-21). bwpat, Nr. 4.

Cohen, J. (1992). A power primer. Psychological Bulletin, 112, 155-159.

Deeley, S. J. (2010). Service-learning: thinking outside the box. Active Learning in higher Education, 11, 43-53.

Deming, W.E. (1989). Out of the crisis. Cambridge: Massachusetts Institute of Technology.

Dewey, J. (1915). The school and society. Chicago: University of Chicago Press.

Dewey, J. (1966). Democracy and education. New York: Free Press.

Edelson, D.C. (2002). Design research: what we learn when we engage in design. The Journal of the Learning Sciences, 11(1), 105-121.

Euler, D. (2005). Forschendes Lernen. In S. Spoun \& W. Wunderlich (Hrsg.), Studienziel Persönlichkeit (S. 253-272). Frankfurt a.M.: Campus.

Field, A., Miles, J., \& Field, Z. (2012). Discovering statistics using R. Los Angeles, London, New Delhi, Singapore, Washington DC: SAGE.

Flick, U. (2010). Qualitative Sozialforschung (3. Aufl.). Reinbek: Rowohlt.

Gerholz, K.-H., \& Losch, S. (2015). Can service learning foster a social responsibility among students? In L. O'Riodan, S. Heinemann \& P. Zmuda (Hrsg.), New perspectives on corporate social responsibility (S. 602-622). Wiesbaden: Springer.

Gerholz, K.-H., Liszt, V., \& Klingsieck, K.B. (2015). Didaktische Gestaltung von Service-Learning (S. 1-18). bwpat Nr. 28

Godfrey, P.C., Illes, L. M., \& Berry, G. R. (2005). Creating breath in business education through servicelearning. Academy of Management Learning \& Education, 4(3), 309-323.

Jacoby, B. (2009). Civic engagement in today's higher education. In B. Jacoby (Hrsg.), Civic engagement in higher education (S. 5-30). San Franciso: Jossey-Bass.

Klafki, W. (1985). Neue Studien zur Bildungstheorie und Didaktik. Weinheim: Beltz.

Kuckartz, U. (2012). Qualitative Inhaltsanalyse. Methoden, Praxis, Computerunterstützung. Weinheim: Beltz.

Kuckartz, U. (2014). Mixed Methods. Methodologie, Forschungsdesigns und Analyseverfahren. Wiesbaden: VS. 
Mabry, J. B. (1998). Pedagogical variations in service-learning and student outcomes. Michigan Journal Of Community Service-Learning, 5, 32-47.

Mayring, P. (2002). Einführung in die qualitative Sozialforschung (5. Aufl.). Weinheim, Basel: Beltz.

Peterßen, W.H. (2009). Kleines Methoden-Lexikon (3. Aufl.). München: Oldenbourg.

Plomp, T. (2007). Educational design research: an introduction. In T. Plomp \& N. Nieveen (Hrsg.), An introduction to educational design research (S. 9-36). Enschede: SLO.

Reetz, L. (1999). Zum Zusammenhang von Schlüsselqualifikationen - Kompetenzen - Bildung. In T. Tramm, D. Sembill, F. Klauser \& E. G. John (Hrsg.), Professionalisierung kaufmännischer Berufsbildung (S. 32-51). Frankfurt a.M.: Peter Lang.

Reigeluth, C.M., \& Frick, T.W. (1999). Formative research: a methodology for creating and improving design theories. In C.M. Reigeluth (Hrsg.), Instructional design theories and models (S. 663-651). Mahwah: Erlbaum.

Reinders, H., \& Hildebrandt, J. (2008). servU - Service-Learning an Universitäten. Dokumentation des Längsschnitts WS 2007-08. Schriftenreihe Empirische Bildungsforschung, Bd. 5. Würzburg: Universität Würzburg.

Reinders, H., \& Wittek, R. (2009). Persönlichkeitsentwicklung durch Service-Learning an Universitäten. In K. Altenschmidt, J. Miller \& W. Stark (Hrsg.), Raus aus dem Elfenbeinturm? (S. 128-143). Weinheim, Basel: Beltz.

Reinmann, G. (2005). Innovation ohne Forschung? Unterrichtswissenschaft, 33(1), 52-69.

Ritzmann, S., Hagemann, V., \& Kluge, A. (2014). The Training Evaluation Inventory (TEI). Vocations and Learning, 7(1), 41-73.

Schwarzer, R., \& Jerusalem, M. (Hrsg.). (1999). Skalen zur Erfassung von Lehrer- und Schülermerkmalen. Dokumentation der psychometrischen Verfahren im Rahmen der Wissenschaftlichen Begleitung des Modellversuchs Selbstwirksame Schulen. Berlin: Freie Universität Berlin.

Slepcevic, P., \& Stock, M. (2009). Selbstverständnis der Wirtschaftspädagogik in Österreich und dessen Auswirkungen auf die Studienplanentwicklung am Standort Graz (S. 1-20). bwpat Nr. 16.

Slepcevic-Zach, P. (2017). Service-Learning in der wissenschaftlichen Berufsvorbildung von Wirtschaftspädagog/inn/en. Zeitschrift für Berufs- und Wirtschaftspädagogik, 113(2), 303-324.

Slepcevic-Zach, P., \& Gerholz, K.-H. (2015). Service-Learning - Entstehung, Wirksamkeit und konkrete Umsetzung. In E. Augustin, M. Hohenwarter, G. Salmhofer \& L. Scheer (Hrsg.), Theorie, die ankommt. Grazer Beiträge zur Hochschullehre (Bd. 6, S. 61-76). Graz: Leykam.

Sliwka, A., \& Frank, S. (2004). Service-Learning. Verantwortung lernen in Schule und Gemeinde. Weinheim: Beltz.

Tafner, G., Stock, M., \& Slepcevic-Zach, P. (2013). Die Wirtschaftspädagogik als Disziplin. In M. Stock, P. Slepcevic-Zach \& G. Tafner (Hrsg.), Wirtschaftspädagogik. Ein Lehrbuch (S. 1-118). Graz: UniPress.

Zehetner, E., Stock, M., \& Slepcevic-Zach, P. (2016). Wipäd - und dann? Ergebnisse aus der aktuellen Abso-Befragung am Standort Graz. Wissenplus Wissenschaft, 3-15(16), 30-33. 